\title{
FDA Structured Product Labeling Terminology
}

National Cancer Institute

\section{Source}

National Cancer Institute. FDA Structured Product Labeling Terminology. NCI Thesaurus.

Code C54452.

Terminology used in the framework of the Structured Product Labeling documents. 